\title{
Success in Inducing Effective Rooting in Triticale $x$ Wheat and Wheat $x$ Wheat Derived Haploid Plantlets in Cocopeat Mixture
}

\author{
Navdeep S. Jamwal ${ }^{1 *}$, Harinder K. Chaudhary ${ }^{1}$, Anila Badiyal ${ }^{1}$, Waseem Hussain ${ }^{2}$, \\ Pankaj Katoch ${ }^{1}$ and Nimit Kumar ${ }^{1}$
}

\author{
${ }^{1}$ Molecular Cytogenetics and Tissue Culture Lab., Department of Crop Improvement, CSK HP \\ Agricultural University, Palampur-176062, India \\ ${ }^{2}$ Department of Agronomy and Horticulture, University of Nebraska \\ Lincoln-68588-0915, USA \\ *Corresponding author
}

\section{A B S T R A C T}

\begin{tabular}{|l|}
\hline K e y w o r d s \\
Triticale, Wheat, Haploid, \\
Liquid MS medium, \\
Cocopeat, Perlite, \\
Vermiculite, Roots
\end{tabular}

\section{Introduction}

Doubled haploidy (DH) offers an opportunity to speed up traditional breeding methods. It has increased the pace of wheat improvement programmes through rapid attainment of homozygosity, resulting in acceleration of varietal developmental programmes which otherwise can take 7-8 years (Chaudhary et al., 2015). Though the technique itself is an accelerating force for crop improvement endeavours but it is limited by the low number of haploids recovered. Breeders across the world used several DH techniques that is anther culture, ovary culture, microspore culture, ovule culture and chromosome elimination techniques including bulbosum technique (Barclay 1975) and wheat $x$ maize system (Laurie and Bennett 1988). Gamete culture techniques have found limited applications owing to their genotypic specific response and several culture generated 
problems. Like gamete culture, bulbosum technique has also very narrow range of responsive wheat genotypes to haploid induction. But wheat $\mathrm{x}$ maize system has also been utilized across the globe being genotype non-specific, yet, it could not draw positive results in wheat-rye and triticale-wheat hybrids. The inquisitive search for more responsive pollinators for chromosome elimination system led Chaudhary et al., (2005) to invent a dynamic Imperata cylindrica-mediated chromosome elimination technique which has not only shown overwhelming success over wheat $\mathrm{x}$ maize system in wheat-wheat hybrids (Mayel et al., 2016), but has also recorded a huge success in wheat-rye (Kishore et al., 2012) and triticalewheat hybrids (Badiyal et al., 2014; Jamwal et al., 2016) and durum wheat (Mahato and Chaudhary 2015). Thus, I. cylindrica mediated system has emerged as an efficient alternative to wheat $\mathrm{x}$ maize system for $\mathrm{DH}$ breeding (Chaudhary et al., 2005; Pratap et al., 2005).

The efficiency of doubled haploidy breeding critically depends on the tissue culture and plant regeneration techniques (Chaudhary et al., 2015). Tissue culture techniques are an integral part of doubled haploidy breeding process as these provide opportunity to rescue underdeveloped embryo produced through wide hybridization which otherwise could have starved to death. Hence, a number of studies have been focused on in vitro culture, regeneration and haploid plants. For years, Murashige and Skoog (MS) medium (1962) has been utilized across the globe for effective regeneration of the haploid embryo cultures in wheat and MS medium has been reported apt for the embryo germination (Singh et al., 2005; Puja et al., 2011). Researchers have been refining the $\mathrm{DH}$ protocols continuously to simplify the methods, to make the technology economical and to enhance the efficiency of haploid production (Tadesse et al., 2013). If a suitable protocol for plant regeneration is available, wheat research can take huge leap for successful production of large number of doubled haploids. But the proper regeneration and growth of the plant is dependent on the vigour of the embryo as well as the physiology of the plant system. Since small embryos fail to germinate and only embryos with good size germinate (Niroula $e t$ al., 2007) hence, after germination, absorption and proper delivery of the nutrients to the growing parts of the plant is necessary for attaining healthy plants, which is in turn ensured by roots. Weak root system of haploid plants makes them more vulnerable to hardening stresses resulting in high mortality of plants after regeneration. The establishment of protocols for vigorous root development using various rooting media depending on the specific requirements of the crops is of utmost importance. Well proliferated roots have an immense role in establishment, anchorage and nutrition uptake. Poor efficiency of agar medium for wheat root proliferation was reported by Kudrika et al., (2012). Hence, for good initiation of roots, half strength MS medium is widely used. Liquid rooting medium devoid of sucrose, consisting of half strength MS salts, 1mg naphthalene acetic acid per litre (NAA) and 1mg indole-3-butyric acid (IBA) per litre have been used for inducing profuse rooting in regenerated plants (Chaudhary et al., 2005; Ayed et al., 2011). Observation for four-five years revealed that liquid media has not been efficiently serving the purpose for profuse rooting in haploid wheat plants, which led the authors search for more alternatives.

Since the last few years cocopeat is used alone or in combination with other materials like perlite, vermiculite, sand gravel etc. in hardening of tissue culture plants, hydroponics, horticultural and forest nurseries (Awang et al., 2009; Bhardwaj 2014). It has high total pore space, high water content, low shrinkage, low bulk density and slow biodegradation along with Potassium and sodium which provide adequate nutrition to 
roots (Evans et al., 1996; Prasad, 1997). This media is being used for hardening the haploid plants but not for rooting. In case of wheat haploid regeneration, no study has been reported regarding the comparative performance of liquid media and cocopeat mixture for rooting enhancement. Keeping this in view, the present study was aimed to assess the effect of half strength MS media and CPV mixture (3 parts coco-peat: 1 part perlite: 1 part vermiculite) on root traits.

\section{Materials and Methods}

The experiment was conducted at Molecular Cytogenetics and Tissue Culture Lab, CSK, HP Agricultural University, Palampur, India. The experimental material consisted of haploid plantlets derived from $F_{2}, F_{3}$ and $F_{4}$ generations of triticale $\mathrm{x}$ wheat and wheat $\mathrm{x}$ wheat crosses following standardized haploid production protocol for wheat and triticale using Imperata cylindrica -mediated chromosome elimination approach developed by Chaudhary et al., (2005) and Pratap et al., (2005), respectively. The pseudo seeds from different crosses were thoroughly sorted against light for embryos. The embryo carrying pseudo seeds were excised under strict aseptic conditions and embryos were cultured on MS medium supplemented with $0.5 \mathrm{mg} / \mathrm{L}$ kinetin, $20 \mathrm{mg} / \mathrm{L}$ each of L-arginine, L-cysteine and L-leucine, $30 \mathrm{~g} / \mathrm{L}$ sucrose and $8 \mathrm{~g} / \mathrm{L}$ agar agar. The cultured embryos were incubated in the dark at $20 \pm 2^{0} \mathrm{C}$ for regeneration. The regenerated plantlets were then placed in the growth room at $20 \pm 2^{\circ} \mathrm{C}$ with 10/14 hours light/dark regime until they developed sufficiently. Subsequently, half of the haploid regenerants were transferred to liquid rooting medium (1/2 strength MS salts, no sucrose and $1 \mathrm{mg} / \mathrm{l}$ each of $\alpha$-naphthalene acetic acid and Indole 3-butyric acid) in order to induce profuse rooting in the haploid plantlets. Rest half of the haploid plantlets were transferred to rooting mixture consisting of cocopeat, perlite and vermiculite (CPV) in the ratio of 3:1:1. The haploid plantlets were kept in the both type of media for two weeks. Three plants of each cross per generation per treatment were used for data recording. After two weeks, plants were taken out and data were recorded for root characters viz., root length $(\mathrm{cm})$, number of roots originating from crown portion of the plant and root area $\left(\mathrm{cm}^{2}\right)$. Root length was measured using centimeter scale ruler while for surface area, EVP scanner with WINFOLIA PRO SOFTWARE (Reagent Industries, Toronto, Canada) software was used. Variance among different root traits and generations included in the study was analyzed using two way factorial design using statistical package SPSS 16.0 (Statistical Package for Social Sciences, SPSS Inc. Chicago, IL). The significance of difference among the parameters i.e. root length $(\mathrm{cm})$, root area $\left(\mathrm{cm}^{2}\right)$ and number of roots in liquid medium and CPV medium was analyzed using student's $t$ test. Pearson's correlation coefficient was derived using Panse and Sukhatme (1984). After recording the root traits, the haploid plants were subjected to $(0.1 \%$ colchicine solution $+1.5 \%$ DMSO $)$ for six hours and transplanted into pots containing soil, sand and vermi-compost (2:1:1). Soil and sand were autoclaved at $15 \mathrm{psi}$ for $15 \mathrm{~min}$. The survival of plants was recorded after two week of colchicine application.

\section{Results and Discussion}

In production of doubled haploids, major hurdle is the regeneration of haploid plants. Well developed rooting system can help in absorption of water and assimilation of the nutrients from the growth medium as well as provide proper anchorage which ultimately contributes in proper growth and development of the haploid plantlets. Hence, induction of profuse rooting using rooting medium is an integral part of the tissue culture process for efficient haploid formation. Liquid half 
strength MS medium is commonly used rooting medium worldwide (Chaudhary et al., 2005; Ayed et al., 2011) whereas, cocopeat alone or in combination with other particulate media i.e., perlite and vermiculite is used for hardening of tissue culture plants. This led the authors to search for another potential medium which can fulfill the all the aforesaid mentioned requirements. Since many years cocopeat, perlite and vermiculite (CPV) have been used in the ratio of 3:1:1 in tissue culture labs for hardening purpose only, not for rooting. Hence, in the present study, both CPV as well as half strength MS media were assessed and compared for their performance towards rooting in haploids.

To study the effect of different generations on the performance of media, segregating generations viz., $\mathrm{F}_{2}, \mathrm{~F}_{3}$, and $\mathrm{F}_{4}$ of triticale $\mathrm{x}$ wheat and wheat $\mathrm{x}$ wheat derivatives were included. In present investigation analysis of variance revealed significant difference among both rooting media for all three parameters for root morphology (root length, root surface area and number of roots), depicting the effect of media on recorded traits whereas the same was found to be non-significant for triticale $\mathrm{x}$ wheat and wheat $x$ wheat crosses. The effect of the parentage of triticale $\mathrm{x}$ wheat and wheat $\mathrm{x}$ wheat crosses were found to be similar towards all the traits under observation. The effect of different generation's viz., $\mathrm{F}_{2}, \mathrm{~F}_{3}$, and $\mathrm{F}_{4}$ of triticale $\mathrm{x}$ wheat and wheat $\mathrm{x}$ wheat derivatives on the performance of media was also analyzed and observed to be nonsignificant. The study ruled out the segregational effect on the root characteristics based on the observations in both media. Moreover, different crosses did not vary significantly for the root growth parameters. Hence, a direct relation can be derived between the media and root traits.

The root length is considered to be an important trait because it reveals the penetration of roots in soil and is a direct measure for mineral and solutes uptake efficiency (Manschadi et al., 2006). In the present investigation, among the plants grown in liquid medium, the root length varied from $12.57 \mathrm{~cm}$ (DT123 x DH40) to $16.50 \mathrm{~cm}$ (DH52 $x$ DH40) with an average of $14.54 \mathrm{~cm}$. Whereas, in CPV medium minimum and maximum root length was observed to be $18.57 \mathrm{~cm}$ (DH5 x DH40) and $26.77 \mathrm{~cm}$ (HPW236 x C306), respectively with an average of $22.04 \mathrm{~cm}$. The root length of the plants grown in CPV medium was found to be doubled to that of liquid medium. This may be due to the congenial rhizosphere provided by CPV medium. Though the medium is solid and imitates the soil conditions yet it provides least obstruction to the growing roots. On the other side, root growth in liquid medium is not restricted by any physical barrier (Awang et al.2009). Besides, half strength of nutrient in liquid media force the plant roots to grow more and fast so as to get enough nutrition for the plant. But lack of proper aeration to the growing and continuously respiring root cells leads plant to the state of hypoxia. Low oxygen in the cells leads to anaerobic respiration which causes slow death of new cells thus slowing down the plant growth and development (Liao and Lin, 2001).

Continuous shaking can be a solution to the lack of aeration but can't be applied when the plants are grown in a limited containment such as test tubes. It has also been observed during the investigation that the growth of root in liquid media was not as vigorous as desired which resulted in death of haploid plants while hardening. This is further supported by Mazri, 2012 who observed non-significant deviation in root length in different compositions of liquid MS media. In contradiction, Bhardwaj (2014) observed significant deviation in same parameter when different medium used with and without cocopeat. 
The other root trait which critically influences the anchoring capacity as well as the absorption efficiency of plant is its total surface area. The establishment of a plant depends on its ability to stand upright so as to increase its lodging resistance (Ennos et al., 1993; Crook and Ennos, 1993).

A lot of variation was noticed in the surface area of the roots of haploid plantlets under observation. In liquid medium, it ranged from $2.89 \mathrm{~cm}^{2}$ (DH114 $\mathrm{x}$ DH100) to $7.34 \mathrm{~cm}^{2}$ (DT126 $x$ HPW155) with an average of $5.53 \mathrm{~cm}^{2}$ whereas, the mean value for same trait in CPV medium was found to be $21.14 \mathrm{~cm}^{2}$ with the range $17.00 \mathrm{~cm}^{2}$ (DH114 $\mathrm{x}$ DH100) to $28.60 \mathrm{~cm}^{2}$ (HPW236 x C306). The total surface area of roots was found to be increased four times in CPV medium as compare to MS liquid medium. The root hairs were not well developed in the liquid medium because roots were continuously submerged in liquid leads to anaerobic respiration resulting in the synthesis of alcohol.

Accumulation of alcohol in the growth medium can lead to slow death of new cells which in turn may slow down the plant growth and development (Vartapetian and Jackson, 1997). In CPV medium vermiculite allowed proper drainage and air circulation. Perlite is neutral in $\mathrm{pH}$ and contains air pockets which provide rock sponge for roots (Awang et al., 2009).

The number of roots originating from crown is also influenced by the conditions in which plant is growing. More the accessory roots, more is the strength provided to plant. A positive relation of shoot vigor to the root number has been derived by Richards and Lukacs (2002). In present investigation average number of roots originating from the crown portion was 4.87 in liquid media and 7.47 in CPV media. Out of ten crosses in the experiment, majority of the plants showed less than five roots per plant when grown in the liquid medium whereas in CPV medium, root number was found to be either equal or more than seven in almost all of the plants. In the plants grown in liquid medium, minimum root number was found to be four while in CPV medium, it was seven. A maximum of six roots were observed to be originated from the crown in the liquid medium reared plants in comparison to ten roots in the latter. The results from the present study are completely in accordance with earlier reports showing very less number of roots in plants growing in half strength liquid medium (Mazri 2012). The liquid half strength medium is not as conducive as CPV medium for root formation (Table 1-3).

Table.1 Analysis of variance of rooting characters using different rooting media

\begin{tabular}{|l|l|l|l|l|l|} 
S.no. & Source & df & \multicolumn{3}{|c|}{ Mean sum of squares } \\
\cline { 4 - 6 } & & & Root length & Root area & Root number \\
\hline $\mathbf{1}$ & Replication & 2 & 17.37 & 7.16 & 3.27 \\
\hline $\mathbf{2}$ & Treatment & 19 & $60.23 * *$ & $218.15^{* *}$ & $7.35^{* *}$ \\
\hline $\mathbf{3}$ & Media & 1 & $837.01 * *$ & $3782.17 * *$ & $101.40^{* *}$ \\
\hline $\mathbf{4}$ & Crosses & 9 & 25.27 & 21.12 & 2.52 \\
\hline $\mathbf{5}$ & Interaction & 9 & 8.88 & 19.18 & 1.73 \\
\hline $\mathbf{6}$ & Error & 38 & 19.23 & 8.26 & 1.69 \\
\hline
\end{tabular}

$* \mathrm{P} \geq 0.05 ; * * \mathrm{P} \geq 0.01$ 
Table.2 Effect of media on haploid plants developed from segregating generations of triticale $\mathrm{x}$ wheat and wheat $\mathrm{x}$ wheat crosses on root characters

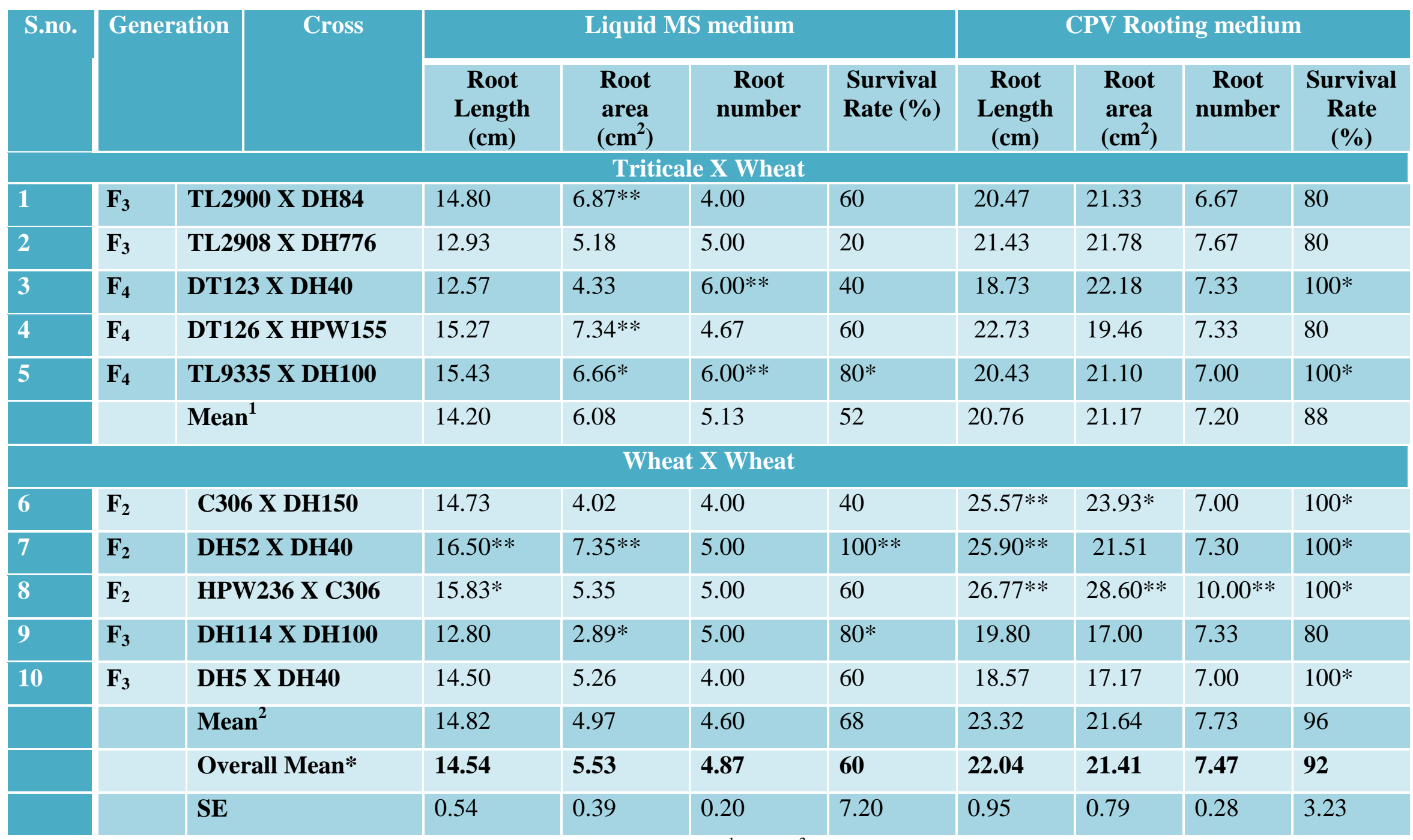

*P $\geq 0.05 ; * * \mathrm{P} \geq 0.01$; significance was tested on overall mean; Overall Mean- Mean ${ }^{1}+$ Mean $^{2}$ 
Table.3 Correlation coefficient among root traits in MS liquid and CPV media

\begin{tabular}{|l|l|l|}
\hline Liquid media & Root number & Root area \\
\hline Root Length & -0.193 & $0.721^{*}$ \\
\hline Root area & -0.023 & \\
\hline CPV media & & $0.709^{*}$ \\
\hline Root Length & $0.539^{*}$ & \\
\hline Root area & $0.716^{*}$ &
\end{tabular}

$* \mathrm{P} \geq 0.05$

Correlation studies help in determining the related performance of traits. This provides information to the researcher about importance of a trait. In the present investigation, contradictory results have been observed for correlation of root traits in both media used. In liquid medium root length ($0.193)$ and root surface area $(-0.023)$ were found to be negatively but non-significantly correlated with root number which depicted that medium was not proficient for more new root formation. A significantly positive association of root number with root length $(0.539)$ and root surface area (0.716) was observed in CPV medium proving its rooting efficiency. These results are completely in agreement with Erayman et al., 2006 who revealed the positive correlation among root length and root number. Similarly Narayanan et al.2014 revealed positive correlation of root length with root surface area in wheat. Besides, Awan et al., 2007, Khan et al., 2013 observed that root length was positively correlated with most of the root traits.

Wide variation was observed in the survival rate of plants after colchicine application which ranged between 20 to 100 per cent. Mortality rate of the plants taken out from liquid medium ranged from 20 to 80 per cent as compare to 80 to 100 per cent survival of plants grown in CPV medium. The present investigation revealed a strong relationship between medium for rooting and plant recovery from colchicine stress, emphasizing the importance of well developed root system in plant growth. CPV medium being porous and soil like provided the natural habitat to the plant root system thus enhancing the survival during acclimatization in the soil.

Conclusively, medium plays a crucial role in primary establishment of haploid plants by providing nourishment for well proliferating root system. By comparing both media under experimentation, CPV (cocopeat: perlite: vermiculte) was proved to be better alternative to the half strength liquid medium for profuse rooting. This study will have far reaching implications for conducting cytological investigations of the triticale $\mathrm{x}$ wheat and wheat $\mathrm{x}$ wheat derived haploid regenerants and also to recover more doubled haploids after colchicine application.

\section{References}

Awan, S.I., S. Niaz, M. Faisal, A. Malik and Ali S. 2007. Analysis of variability and relationship among seedling traits and plant height in semi-dwarf wheat (Triticum aestivum L.). J. Agri \& Social Sci 3: 59-62.

Awang, Y., A.S. Shaharom, R.B. Mohamad and Selamat A. 2009. Chemical and physical characteristics of cocopeatbased media mixtures and their effects on the growth and development of Celosia cristata. American J Agric. \& Bio. Sci. 4: 63-71.

Ayed, S., O. Slama-Ayed, S.J.A. Teixeira, and Slim-Amara, H. 2011. Effect of 
different factors on haploid production through embryo rescue in durum wheat $\times$ maize crosses. Int. J. Plant Breed. 5: $118-121$

Badiyal, A., H. K. Chaudhary, N. S. Jamwal, W. Hussain, A. Mahato and Bhatt, A. K. 2014. Interactive genotypic influence of triticale and wheat on their crossability and haploid induction under varied agroclimatic regimes. Cereal Res. Commun. 42: 700-709.

Barclay, I. R., 1975. High frequencies of haploid production in wheat (Triticum aestivum) by chromosome elimination. Nature. 256: 410-411.

Bhardwaj, R.L., 2014. Effect of growing media on seed germination and seedling growth of papaya cv. 'Red lady'. Afr. J. Pl. Sci. 8: 178-184.

Chaudhary, H.K. A. Badiyal, and Jamwal N.S. 2015. New frontiers in doubled haploidy breeding in wheat. Agricultural Research Journal 52(4): 112.

Chaudhary, H.K., G.S. Sethi, S. Singh, A. Pratap, and Sharma, S. 2005: Efficient haploid induction in wheat by using pollen of Imperata cylindrica. Plant Breed. 124: 96-98.

Crook, M. J., and Ennos, A. R. 1993. The mechanism of root lodging in winter wheat Triticum aestivum L. Journal of Experimental Biology. 44: 1219-1224.

De Kreij, C., and Leeuven, G.J.L. 2001. Growth of pot plants in treated coir dust as compared to peat. Commun. Soil Sci. Plant Anal. 32: 2255-2265.

El-Hennawy, M.A., A.F. Abdalla, S.A. Shafey, and Al-Ashkar, I.M. 2011. Production of doubled haploid wheat lines (Triticum aestivum L.) using anther culture technique. Annals of Agri. Sci. 56: 63-72.

Erayman, M., B. Abeyo, P. Baenziger, H. Budak and Eskridge, K. 2006. Evaluation of seedling characteristics of wheat (Triticum aestivum L.) through canonical correlation analysis. Cereal Res. Commun. 34: 1231-1238.

Evans, M.R., S. Konduru and Stamps, R.H. 1996. Source variation in physical and chemical properties of coconut coir dust. Hort. Science 31: 965-967.

Jamwal, N.S., H.K. Chaudhary, A. Badiyal and Hussain W. 2016. Factors influencing crossability among triticale and wheat and its subsequent effect along with hybrid necrosis on haploid induction. Acta Agriculturae Scandinavica, Section B-Soil \& Plant Science 66: 282-289.

Kishore, N., H.K. Chaudhary, R.K. Chahota, V. Kumar, S.P. Sood and Jeberson, S. 2011. Relative efficiency of the maize and Imperata cylindrica mediated chromosome elimination approaches for induction of haploids of wheat-rye derivatives. Plant Breed. 130: 192-194.

Kudirka, D.T., G.W. Schaeffer and Baenziger P.S. 2012. Wheat: Genetic variability through anther culture. In: Bajaj YPS editor, Biotechnology in agriculture and forestry 2 Crop I Springer Publisher New York p. 39-55.

Laurie, D.A., and Bennett, M.D. 1988. The production of haploid wheat plants from wheat $\times$ maize crosses. Theor. Appl. Genet. 76: 393-397.

Liao, C.T., and Lin, C.H. 2001. Physiological adaptation of crop plants to flooding stress. Proc. Natl. Sci. Counc. 25: 148157.

Machii, H., H. Mizuno, T. Hirabayashi, H. Li and Hagio, T. 1998. Screening wheat cultivars for high callus induction and regeneration capability from anther and immature embryo cultures. Plant Cell, Tiss. \& Org. Cult. 53: 67-74.

Mahato, A., and Chaudhary, H.K. 2015. Relative efficiency of maize and Imperata cylindrica for haploid 
induction in durum wheat. Plant Breed. 134: 379-383

Manschadi, A.M., J. Christopher, P. deVoil and Hammer, G.L. 2006. The role of root architectural traits in adaptation of wheat to water-limited environments. Funct. Plant Biol. 33: 823-837.

Mayel, A., H.K. Chaudhary, A. Badiyal and Jamwal N.S. 2016. Comparative pollination efficiency of freshly harvested pollen of Imperata cylindrica and Zea mays for haploid induction in bread wheat. Cereal Research Communications. 44: 162-171

Mazri, M.A., 2012. Effect of liquid media and in vitro pre-acclimatization stage on shoot elongation and acclimatization of date palm (Phoenix dactylifera L.) cv. Najda. Journal of Ornamental Plants 2: 225-231.

Murashige, T., and Skoog, F. 1962. A revised medium for rapid growth and bioassays with tobacco tissue cultures. Physiol. Plant 15: 473-497.

Narayanan, S., A. Mohan, K.S. Gill, and Prasad, P.V.V. 2014. Variability of root traits in spring wheat germplasm. PLoS ONE 9: 100317

Panse, V.G., and Sukhatme, P.V. 1984. Statistical methods for agricultural workers (third ed.) ICAR, New Delhi, India.
Prasad, M., 1997. Physical, chemical and biological properties of coir dust. Acta Hort. 450: 21-29.

Pratap, A., G.S. Sethi and Chaudhary, H.K. 2005. Relative efficiency of different Gramineae genera for haploid induction in triticale and triticale $\times$ wheat hybrids through chromosome elimination technique. Plant Breed. 124: 147-153.

Puja, R.S. Gill, S. Kumar, G.S. Mahal and Bains, N.S. 2011. Effect of growth hormones on caryopses formation and plant regeneration frequency in durum wheat $x$ maize crosses. Journal of Wheat Research 3: 63-68.

Richards, R.A., and Lukacs, Z. 2002. Seedling vigour in wheat - sources of variation for genetic and agronomic improvement. Australian J. Agric Sci., 53: 41-50.

Singh, N., R.K. Behl and Punia, M.S. 2005. Effect of genotypic background on haploid production through embryo rescue in wheat $\times$ maize crosses. Plant Soil Environment 51: 193-196.

Tadesse, W., S. Tawkaz, M.N. Inagaki, E. Picard and Baum, M. 2013. Methods and applications of doubled haploid technology in wheat breeding. ICARDA, Aleppo, Syria. Pp 36.

Vartapetian, B.B., and Jackson, M.B. 1997. Plant adaptation to anerobic stress. Annals of Botany 79: 3-20.

\section{How to cite this article:}

Navdeep S. Jamwal, Harinder K. Chaudhary, Anila Badiyal, Waseem Hussain, Pankaj Katoch and Nimit Kumar. 2018. Success in Inducing Effective Rooting in Triticale $\mathrm{x}$ Wheat and Wheat x Wheat Derived Haploid Plantlets in Cocopeat Mixture. Int.J.Curr.Microbiol.App.Sci. 7(05): 15-23. doi: https://doi.org/10.20546/ijcmas.2018.705.003 